College Dublin, Dublin, Ireland; ${ }^{2}$ Department of Medical Statistics, Informatics and Health Economics, University of Innsbruck, Innsbruck, Austria; ${ }^{3}$ Heart Failure Unit, St. Vincent's University Hospital, Dublin, Ireland

Introduction This analysis set to investigate the relationship between novel biomarkers of cardiovascular morbidity and mortality with diastolic dysfunction in a primary care cohort at heightened cardiovascular risk.

Methods This is a cross-sectional analysis of 616 participants of the STOP HF study with complete echocardiographic data who have established cardiovascular risk factors and no previously known ventricular dysfunction. Data were also available on medical history, medications, biomarkers of inflammation, lipid, renal and hepatic function and routinely measured clinical parameters. The cohort was categorised into those with and without diastolic dysfunction, omitting those with inconclusive echo data $(n=85)$, leaving a population of $n=531$ for analyses. Preliminary analyses were run separately for both genders to establish univariable associates of diastolic dysfunction taking the presence or absence of diastolic dysfunction as the binary outcome. All co-variates with $\mathrm{p}$-values $\leq .2$ were introduced to forward multivariable logistic regression models to establish the foremost associates of diastolic dysfunction.

Results A high prevalence of diastolic dysfunction (67\%) was observed in the cohort. In males, multivariable associates of diastolic dysfunction [Exponential $\beta$-coefficient (95\% CI); p-value] were younger age [1.132; 1.09 to $1.79:<001]$, the absence of AIIA therapy [2.547; 1.18 to $5.49:<0.02]$ and higher ALP levels [28.813; 1.96 to 424.39: <0.02]. In females, diastolic dysfunction was associated with younger age [1.085; 1.05 to $1.12:<0.001]$ and higher GGT levels [4.838; 1.47 to $15.90:<0.01]$.

Conclusions This analysis demonstrates for the first time that parameters of hepatic function may be coherent indicators of early sub-clinical diastolic dysfunction. In this analysis, their association was superior to that more established risk factors and biomarkers such as BNP in this setting.

\section{P2-50 GGT LEVELS ARE A COHERENT INDICATOR OF CARDIOVASCULAR RISK IN PRIMARY CARE IN BOTH MEN AND WOMEN: RESULTS FROM THE STOP HF STUDY}

doi:10.1136/jech.2011.142976h.85

${ }^{1,2} \mathrm{C}$ Conlon, ${ }^{*} \mathrm{C}$ Kelleher, ${ }^{3} \mathrm{H}$ Ulmer, ${ }^{2}$ Dawkins, ${ }^{2} \mathrm{C}$ O'Loughlin, ${ }^{2} \mathrm{M}$ Ledwidge, ${ }^{2} \mathrm{~K}$ McDonald. ${ }^{1}$ School of Public Health, Physiotherapy and Population Science, University College Dublin, Dublin, Ireland; ${ }^{2}$ Heart Failure Unit, St. Vincent's University Hospital, Dublin, Ireland; ${ }^{3}$ Department of Medical Statistics, Informatics and Health Economics, University of Innsbruck, Innsbruck, Austria

Introduction Gamma-glutamyltransferase (GGT) has been re-established as a marker of cardiovascular risk rather than simply an indicator of liver disease. However, there is little data on the associations between GGT and groups with conventional cardiovascular risk factors in the primary care setting. We sought to examine the factors associated with elevated GGT in an Irish primary care population

Methods We explored the baseline data set of the STOP HF Study, a prospective study of a cohort with defined CV risk factors and no known ventricular dysfunction. To identify multivariable associates of higher GGT, we conducted logistic regression, using GGT above and below the 75th percentile as the binary outcome for males $(49 \mathrm{u} / \mathrm{l})$ and females $(38 \mathrm{u} / \mathrm{l})$.

Results Complete data were available in 879 participants. Multivariable associates of GGT [Exponential $\beta$-coefficient (95\% CI); pvalue] in males were younger age [0.97 (0.96 to 0.99$) ;<0.02]$, higher diastolic blood pressure (BP) $[1.05$ (1.02 to 1.07$) ;<0.001]$ total cholesterol [1.99 (1.19-3.39);<0.001] and HsCRP [2.01 (1.12-3.57);<0.02] and lower urea [0.75 (0.63-0.89); <001] and HDL [0.33 (0.18-0.61); $<0.001]$. In females, higher body mass index [1.08 (1.03-1.13); <0.001] and systolic BP $[1.01(1.00-1.02) ;<0.05]$ and the application of $\beta$-blockers [1.49 (1.27-1.87); <0.02] was associated with higher GGT.

Conclusions We demonstrate that independently and even within its normal ranges, GGT is associated with markers of cardiovascular risk in a primary care population. Particularly in males, GGT appears to be a coherent risk factor associated with incipient underlying disease, in keeping with mechanistic evidence suggesting its role in atherogenesis. GGT measurement is an easily accessible and inexpensive biomarker for cardiovascular risk assessment.

\section{P2-51 HIGH RISK AREAS OF CHD IN A LOW INCIDENCE EUROPEAN COUNTRY}

doi:10.1136/jech.2011.142976h.86

${ }^{1,2} \mathrm{~A}$ I C Ribeiro, ${ }^{*}{ }^{1,2} \mathrm{M}$ de Fátima Pina. ${ }^{1}$ INEB - Instituto de Engenharia Biomédica, Porto, Portugal; ${ }^{2}$ Serviço de Higiene e Epidemiologia da Faculdade de Medicina da Universidade do Porto, Porto, Portugal; ${ }^{3}$ ISPUP - Instituto de Saúde Pública da Universidade do Porto, Porto, Portugal

Introduction Geographical differences in coronary heart disease (CHD) mortality have been widely described, but little is known about the incidence of such disease.

Objective Examine the geographic distribution of CHD hospital admissions from 1997 to 2008, in Continental Portugal, identifying relevant peaks of the disease.

Methods Hospital admissions due CHD (ICD9-CM: 410-414×, $429.2 \times$ ) were obtained from the National Hospital Discharge Register. Age-standardised hospitalisation rates (ASHR) were computed by triennium (excluding 1997-1999, because of losses in georeferencing) for ages 35-74 years, at municipality level. Spatial statistics methods were applied to smooth ASHR and identify spatial clusters. Results were overlaid with a map of climate regions.

Results There were 356119 hospitalisations with CHD as primary or secondary diagnosis, more frequent $(66.7 \%)$ and expensive in men but more fatal and longer in women $(p<0.001)$. Rate ratio (highest: lowest) were 5.2, 5.5 and 4.1 (men) and 9.7, 6.5 and 5.5 (women), respectively in 2000-2002, 2003-2005 and 2006-2008. Moran index of spatial autocorrelation showed moderate degree of spatial dependency $(+0.36,+0.33$ and +0.43 for men, +0.35 , +0.33 and +0.41 for women, in 2000-2002, 2003-2005 and 2006-2008) and spatial clusters were identified. A NortheastSouthwest trend (from lowest to highest values) in ASHR was revealed, coincident with the borderline of the two main climate regions.

Conclusions Accentuated geographic differences in ASHR were observed, although rates remained stable in the study period. Higher fatality and lower cost in hospitalisations of women may reflect gender inequalities in the treatment. Spatial patterns suggested environmental factors are also determinants of $\mathrm{CHD}$.

\section{P2-52 A SOBERING TEXT: DEVELOPING AN INTERVENTION DELIVERED BY MOBILE PHONE TO REDUCE BINGE DRINKING IN DISADVANTAGED YOUNG MEN}

doi:10.1136/jech.2011.142976h.87

I Crombie,* D Falconer, J Coyle, L Irvine. University of Dundee, Dundee, UK

Introduction Disadvantaged men suffer substantial harm from heavy drinking. Effective brief interventions to reduce alcohol 
consumption have been developed for middle-aged men. These were mainly delivered in healthcare settings. Disadvantaged young men seldom attend healthcare. A tailored brief intervention delivered by text message provides a low cost method for accessing this hard to reach group.

Methods Focus groups were conducted with disadvantaged young men to explore the social context of drinking and opportunities for intervention. Empirical evidence and social cognition theories were used to develop a series of text messages and images to reduce binge drinking. These were tested in three further focus groups, including one with female partners of binge drinkers.

Results The focus groups identified cost and personal experience of alcohol harms as opportunities for intervention. Humour was identified as a useful lever for behaviour change. The focus groups also provided useful quotes for text messages. A series of 37 text messages and images were developed within the framework of the Stages of Change model. They incorporated the principles of motivational interviewing and used components of successful behaviour change strategies. To promote interaction, several messages requested a response.

Conclusion This study has shown it is possible to encapsulate the theoretical approaches and the major components of brief interventions in a series of text messages. Focus groups highlighted levers for change and aided the selection of acceptable messages. This approach could be used with other social groups and other adverse health behaviours.

\section{P2-53 YOUNG ADULT LUNG FUNCTION AND CAROTID INTIMA- MEDIAL THICKNESS}

doi:10.1136/jech.2011.142976h.88

B Curry,* A Venn. Menzies Research Institute, University of Tasmania, Hobart, Australia

Introduction Impaired lung function has been associated with an increased risk of cardiovascular disease outcomes, mortality and subclinical disease including increased carotid intima-media thickness (cIMT) and carotid plaques. However few population-based studies have investigated the association between lung function and cIMT in young adults.

Objective To investigate the associations of lung spirometric measures with cIMT in healthy young Australian men and women.

Methods Data were collected from a population-based sample of 872 males and 792 non-pregnant females (aged 26-36 years). The associations of adult Forced Vital Capacity (FVC) and Forced Expiratory Volume $\left(\mathrm{FEV}_{1}\right)$ with cIMT were investigated using partial correlation.

Results The mean cIMT values (SD) were 0.581 (0.099) and 0.553 (0.080) $\mathrm{mm}$ for non-smoking, and 0.575 (0.097) and 0.567 (0.099) $\mathrm{mm}$ for smoking males $(\mathrm{n}=187)$ and females $(\mathrm{n}=161)$ respectively. Significant negative associations of $\operatorname{FEV}_{1}(\rho=-0.100 \mathrm{p}=0.012)$ and FVC $(\rho=-0.087 \mathrm{p}=0.029)$ with cIMT were observed for nonsmoking females after adjustment for age, height, asthma, blood pressure, weight, percentage body fat and fitness. In males there was also a negative association of FVC with cIMT in non-smokers $(\rho=-0.076 \mathrm{p}=0.058)$ and with $\mathrm{FEV}_{1} / \mathrm{FVC}$ in smokers $(\rho=-0.196$ $p=0.009)$. These associations were independent of $C$ reactive protein and blood lipids. No significant associations were observed for female smokers.

Conclusion In this sample of healthy young men and women airway obstruction was associated with higher cIMT in male smokers. However in non-smoking men and women, FVC was also associated with higher cIMT independently of other cardiovascular disease risk factors.

\section{P2-54 DIET AND RISK OF DIVERTICULAR DISEASE IN THE EUROPEAN PROSPECTIVE INVESTIGATION INTO CANCER AND NUTRITION (EPIC)-OXFORD COHORT, A PROSPECTIVE STUDY OF BRITISH VEGETARIANS AND NON-VEGETARIANS}

doi:10.1136/jech.2011.142976h.89

F Crowe, ${ }^{*}$ P Appleby, N Allen, T Key. University of Oxford, Oxford, UK

Introduction Vegetarians and those consuming a high fibre diet are thought to have a lower risk of diverticular disease but there is little evidence from prospective studies to substantiate these associations. The objective was to examine the associations between vegetarianism and dietary fibre intake with the risk of diverticular disease in the EPIC-Oxford study.

Methods This analysis of the EPIC-Oxford cohort included 47033 men and women living in England or Scotland of whom 15459 (33\%) were vegetarians. Diverticular disease cases were identified through linkage with hospital records. The RR of diverticular disease by vegetarian status and quintiles of dietary fibre intake was estimated using multivariate Cox proportional hazards regression models.

Results After an average follow-up of 11.6 years, there were 812 incident cases of diverticular disease. Vegetarians had a $30 \%$ lower

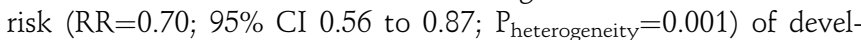
oping diverticular disease compared with non-vegetarians. There was also an inverse association with fibre intake; participants in the highest quintile ( $>25 \mathrm{~g} /$ day) of dietary fibre intake had a $41 \%$ lower risk $\left(\mathrm{RR}=0.59 ; 95 \% \mathrm{CI} 0.46\right.$ to $\left.0.78 ; \mathrm{P}_{\text {trend }}<0.001\right)$ compared to those in the lowest quintile ( $<14 \mathrm{~g} /$ day). After mutual adjustment, both a vegetarian diet $\left(P_{\text {heterogeneity }}=0.015\right)$ and a higher intake of fibre $\left(\mathrm{P}_{\text {trend }}=0.001\right)$ were significantly associated with a lower risk of diverticular disease.

Conclusion Consuming a vegetarian diet and a high intake of dietary fibre were both associated with a lower risk of diverticular disease.

\section{P2-55 INFLUENCE OF SEXUAL MATURATION ON BODY MASS INDEX IN A LONGITUDINAL STUDY WITH ADOLESCENTS FROM BRAZIL}

doi:10.1136/jech.2011.142976h.90

${ }^{1} \mathrm{D}$ Cunha, ${ }^{2} \mathrm{G}$ Veiga, ${ }^{1} \mathrm{R}$ Sichieri. ${ }^{1}$ Institute of Social Medicine, State University of Rio de Janeiro, Rio de Janeiro, Rio de Janeiro, Brazil; '2Department of Nutrition, Federal University of Rio de Janeiro, Rio de Janeiro, Rio de Janeiro, Brazil

Introduction Body mass index (BMI) during adolescence is highly influenced by the pubertal maturation, and early maturation has been associated with overweight, but it is still uncertain if these factors are just a cluster of conditions or whether pubertal maturation influence BMI increments. This study aimed to examine the influence of sexual maturation (SM) on fatness and BMI increments during adolescence among Brazilians aged between 9 and 13 years. Methods 598 girls and 518 boys were followed from 1-year school period. Maturational timing was self-assessed using the Tanner stage ratings (breast development for girls and pubic hair for boys). Early maturation was determined as the age bellow the median age within each gender SM group.

Results Girls with early maturation showed a statistically significant greater prevalence of overweight (WHO classification) compared to those without early maturation $(23.7 \%$ vs $15.4 ; \mathrm{p}=0.02)$ at baseline and similar results were found after 1-year follow-up. On the other hand weight gain during follow-up was not associated to maturation either calculated by stages of sexual maturation, according to Tanner staging or to early maturation, for girls and boys.

Conclusions The results suggest that at baseline and follow-up BMI was highly associated with SM for girls, but BMI increments during follow-up was unrelated to SM for both sexes suggesting a non 